\title{
Rice Cake Ileus
}

\author{
Hiroaki Saito ${ }^{1}$, Tsuyoshi Suda ${ }^{1}$, and Naoki Oishi ${ }^{1}$ \\ ${ }^{1}$ Kanazawa Municipal Hospital
}

May 14, 2020

\begin{abstract}
Rice cake is a traditional Japanese food. Interestingly, rice cake can stay in the intestine due to its stickiness and cause foodinduced ileus. This case shows an example of intestinal obstruction caused by rice cake. Globalization of Japanese food culture necessitates a deeper understanding of such a characteristic disease.
\end{abstract}

\section{Text:}

Mochi is a traditional Japanese rice cake, often eaten during New Year. It is very sticky and difficult to swallow (Figure 1). A 68-year-old woman visited our emergency department complaining of abdominal pain, nausea, and constipation. Physical examination showed mid-abdominal mild tenderness and increased bowel sounds. She revealed having consumed rice cakes the day before. Computed tomography (CT) revealed high-density materials in the small intestine with proximal intestinal dilation (Figure 2). Thus, she was diagnosed with rice cake-induced small bowel ileus. She was admitted and treated conservatively: bed rest, stopping meals, and intravenous fluids. Her symptoms improved within a few days.

Phytobezoar-induced ileus has been reported ${ }^{1}$, however, there are few cases of rice cake-induced small bowel ileus. Rice cake ileus is caused by swallowing rice cakes without adequate chewing, possibly caused by the use of dentures, missing teeth, and weakened mastication ${ }^{2}$. Symptoms are non-specific: abdominal pain, nausea, and vomiting ${ }^{1,2}$. A detailed medical history is important for diagnosis. Rice cakes are not visible on abdominal X-ray images ${ }^{2}$, but abdominal CT can reveal high-density materials. Most cases showed improvement after conservative treatment. Globalization of Japanese food culture necessitates a deeper understanding of such a characteristic disease.

\section{Figure Legends:}

Figure 1.Rice cake is called "mochi" in Japanese. It is very sticky in nature.

Figure 2.Computed tomography revealing small bowl ileus and high-density materials confirmed at the obstruction point.

\section{Conflicts of Interest:}

There are no conflicts of interest to declare.

\section{Source of Funding:}

This research did not receive any specific grant from funding agencies in the public, commercial, or not-forprofit sectors. Thus, no funding source was involved in the study design; collection, analysis, or interpretation of the data; writing of the manuscript; or decision to submit the paper for publication.

\section{Author Contributions:}


HS contributed to the editing of the manuscript and preparation of the figures. cared for the patient, conducted the literature search, edited the manuscript. ON contributed to the editing of the manuscript and provided expert opinion on gastroenterology.

\section{References}

1. Dikicier, E., Altintoprak, F., Ozkan, O.V., Yagmurkaya, O., Uzunoglu, M.Y. 2015. Intestinal obstruction due to phytobezoars: An update. World J Clin Cases 3:721-6.

2. Miura, T., Kimura, N., Nakamura, J., Yamada, S., Miura, T., Yanagi, M., et al. 2011. Rice cake ileus A rare and ethnic but important disease status in East-Southern Asia. Intern Med 50:2737-9.

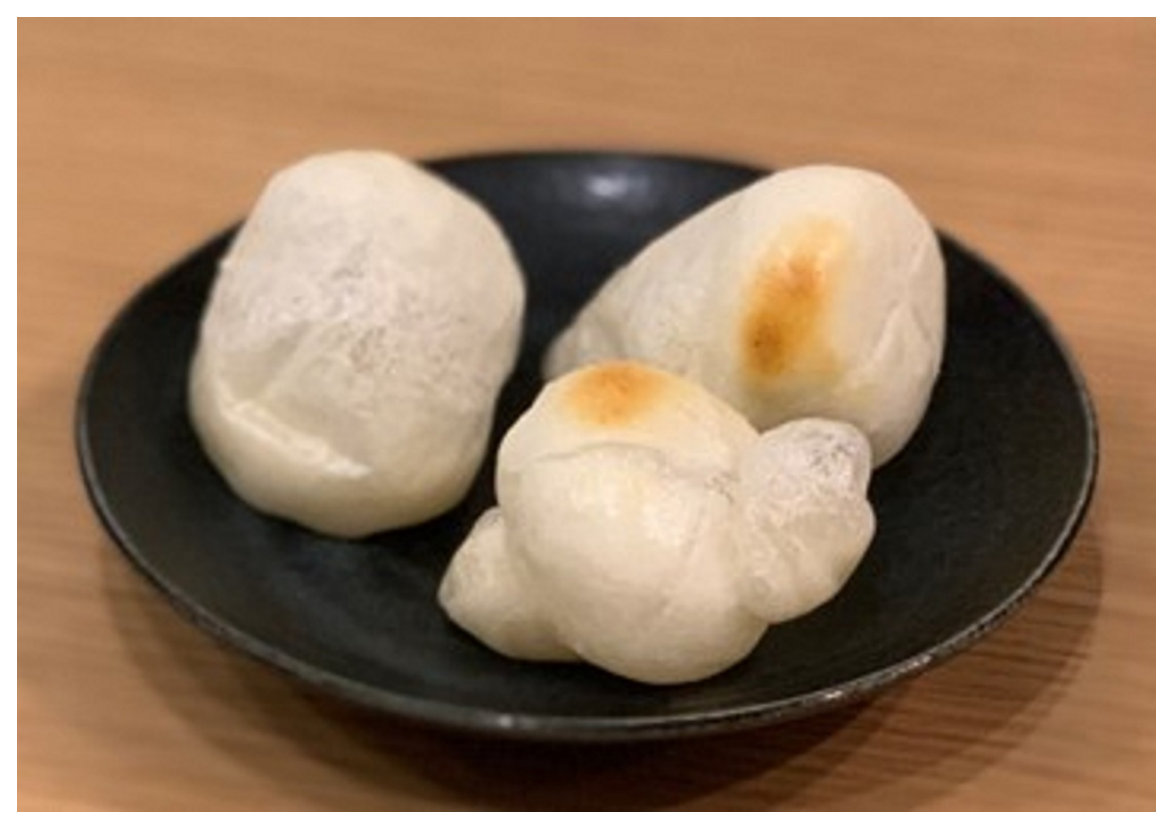




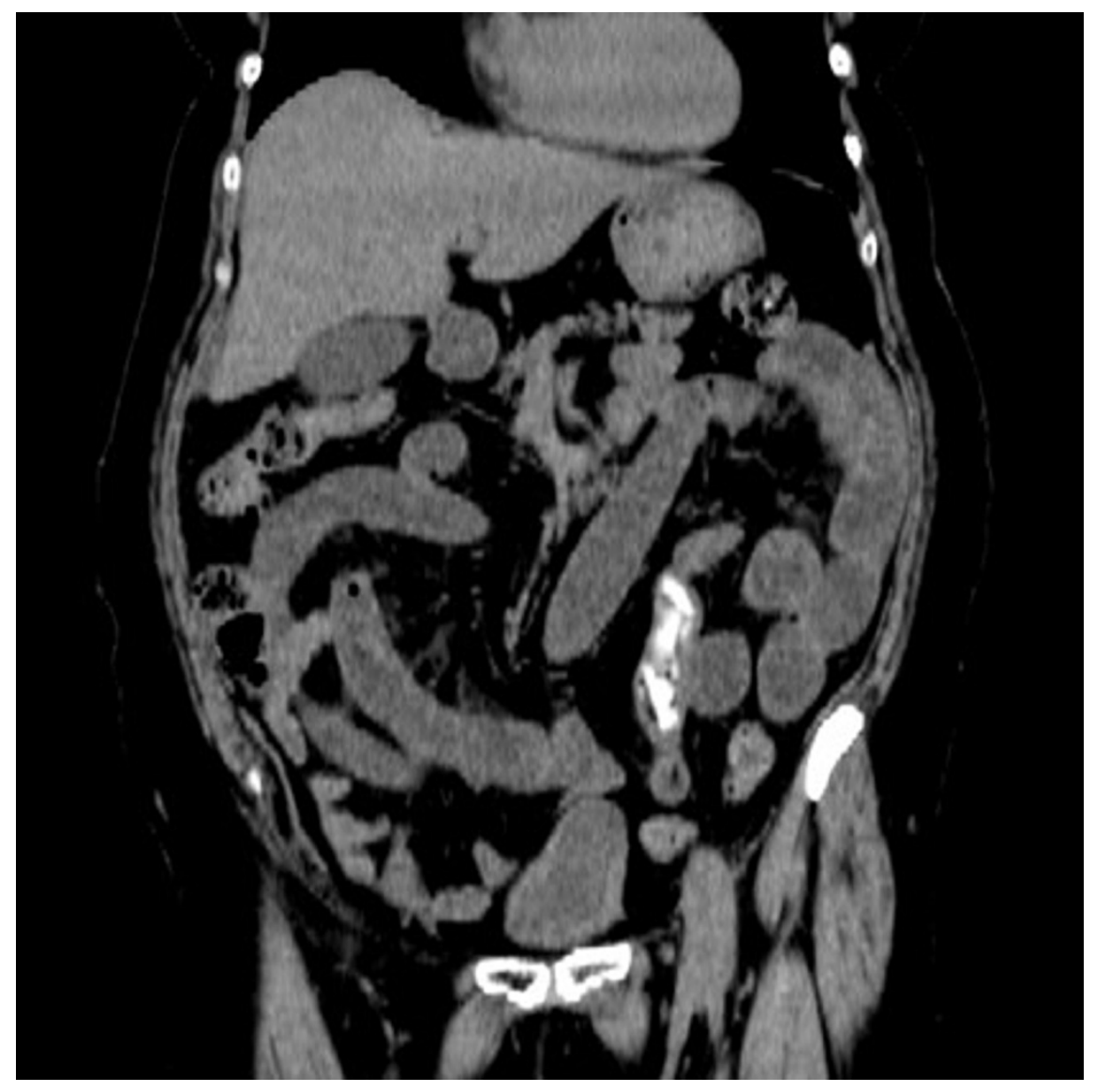

